\title{
Morphology and Elastomeric Properties of Isotactic Polypropylene/ Hydrogenated Polybutadiene Blends
}

\author{
Petr Svoboda, Hiromu SaIto, Tsuneo ChIBA, Takashi Inoue, ${ }^{\dagger}$ and Yasuhiko TaKemura * \\ Dept. of Organic and Polymeric Materials, Tokyo Institute of Technology, \\ 2-12-1, Ookayama, Meguro-ku, Tokyo 152-8552, Japan \\ * TPE Laboratory, JSR Co. Ltd, 100 Kawajiri, Yokkaichi 510-8552, Japan
}

(Received December 16, 1999; Accepted August 1, 2000)

\begin{abstract}
Isotactic polypropylene (PP) was found miscible on a molecular level with hydrogenated polybutadiene (hBR). As there is a possibility of using this blend for designing new types of thermoplastic elastomer (TPE), mechanical properties were measured with the emphasis on elastic behavior. The best elastomeric behavior was found for blends crystallized at room temperature $\left(26^{\circ} \mathrm{C}\right)$, while poor elastomeric properties with high modulus were found for blends crystallized at high temperatures $\left(e . g ., 120^{\circ} \mathrm{C}\right)$. Elastic properties of blends quenched to low temperatures $\left(e . g ., 0^{\circ} \mathrm{C}-\right.$ $-73^{\circ} \mathrm{C}$ ) were slightly worse. Transmission electron microscopy showed that the blend crystallized at the high temperature had a crosshatch lamella structure, while the blends crystallized at lower temperatures (below room temperature) had only small fragmented lamellar crystallites. The latter structure of PP is very unique and can be found only under special conditions. The essential may be the presence of high molecular weight impurity, which prevents formation of crosshatch lamellar structure at the high supercooling depths. Fragmented crystals then probably function as tie points for amorphous chains so that good elastomeric behavior can be achieved. There is a certain possibility of improvement in elastic properties, by annealing quenched samples at high temperature close to melting point of PP. In this case, the crystallinity and size of lamellae increased, while the fragmented character of lamellae was preserved.

KEY WORDS Polypropylene / Hydrogenated Polybutadiene / Blend / Miscible / Thermoplastic Elastomer / Fragmented Lamellar Crystal /
\end{abstract}

One can prepare the polyolefinic thermoplastic elastomer (TPE) by dynamic vulcanization of the blend of polypropylene (PP) with ethylene-propylene rubber (EPDM); e.g., by melt-mixing a 40/60 PP/EPDM blend in the presence of curatives, such as sulphur, accelerators and peroxides. ${ }^{1,2}$ This process yields a two-phase material in which cured EPDM particles with a diameter of a few $\mu \mathrm{m}$ are dispersed in PP matrix. There have been several approaches to explain the elastic behavior of this blend. One is analysis of deformation mechanism of the twophase system by the finite element method (FEM). ${ }^{3}$ In this FEM analysis, the matrix is implicitly assumed to consist of neat PP. However, the matrix may be a mixture of PP and EPDM.

Most pairs of dissimilar polymer are immiscible in a quiescent state. At high shear rates, however, the LCST (lower critical solution temperature) shifts to higher temperatures and thus one-phase mixture is achieved at high shear rates. ${ }^{4-6}$ In polyolefinic blends, Sano et al. found a regularly phase-separated structure with periodic distance of about $150 \mathrm{~nm}$ in injection-moulded blend of PP with high-density polyethylene (PP/HDPE 60/40). This structure can be achieved only by spinodal decomposition mechanism (under zero shear rate in mould) from a single-phase mixture attained (by UCST depression or LCST elevation) in high shear fields in an injection moulding machine (UCST-upper critical solution temperature). ${ }^{7}$

Yang et al. found that the matrix in commercial polyolefinic TPE, Santoprene ${ }^{\circledR}$, is probably composed of PP/ EPDM blend. This implies that the matrix is less ductile and more elastomeric than neat PP. In rubber particles the lamellae of PP were found. Thus, partial miscibility

\footnotetext{
${ }^{\dagger}$ To whom correspondence should be addressed.
}

between those two components seems caused by high shear field during the dynamic vulcanization. ${ }^{8}$

Otsuka et al. found that hydrogenated poly(styreneco-butadiene) is miscible with PP above the melting point of PP even at quiescent condition. This could be a good model blend for the matrix of commercial TPE. However UCST was found at around $100^{\circ} \mathrm{C}$. A two-phase structure is thus formed with periodic distance about 40 $\mathrm{nm}$. This blend shows very good elastomeric properties and can be called as a new type of TPE. However, the structure is still rather complicated, because crystallization is probably affected by spinodal decomposition..$^{9,10}$

Recently we found that PP is completely miscible with hydrogenated polybutadiene ( $\mathrm{hBR}$ ) above the melting point of PP and the UCST or LCST type of phase boundary does not exist. The situation is simpler; i.e., the single-phase melt can be crystallized without liquid-liquid phase separation and crystallized blend could be a nice model system for the matrix of TPE. The aim of this study was at first to find the effects of hBR (high molecular weight impurity) on mechanical properties for blends crystallized at different crystallization temperatures and secondly to find the relationship between mechanical properties and the crystalline morphology. The elastic behavior was tested at tensile test machine by measuring the residual strain after stretching to desired strain. For morphology analysis, transmission electron microscopy (TEM), wide-angle X-Ray diffraction (WAXD) and differential scanning calorimetry (DSC) were conducted.

\section{EXPERIMENTAL}

Isotactic polypropylene (PP) was obtained from Mitsui Chemicals Inc. (J $3 \mathrm{HG}, M_{\mathrm{w}}=3.5 \times 10^{5} \mathrm{~g} \mathrm{~mol}^{-1}$ and $M_{\mathrm{n}}=$ 
$\left.5 \times 10^{4} \mathrm{~g} \mathrm{~mol}^{-1}\right)$. Hydrogenated polybutadiene (hBR) was a special sample prepared by JSR Co. Ltd. $\left(M_{\mathrm{w}}=3 \times 10^{5}\right.$ $\mathrm{g} \mathrm{mol}^{-1}$; ethylene/butene molar ratio $=20 / 80$, degree of hydrogenation $\geq 97 \%$ ).

$\mathrm{PP}$ and hBR were melt-mixed (charge $0.7 \mathrm{~g}$ ) at $200^{\circ} \mathrm{C}$ for $7 \mathrm{~min}$ at $100 \mathrm{rpm}$ in a miniature mixer, Mini-Max Moulder (model CS-183 MMX, Custom Scientific Instruments, Inc.). Three blend ratios were used : $\mathrm{PP} / \mathrm{hBR} 75 /$ $25,50 / 50$, and $25 / 75$ in wt. ratio. The melt-mixed blend was extruded and compression moulded to a thin sheet of $0.2 \mathrm{~mm}$ thickness at $200^{\circ} \mathrm{C}$. The moulded sheet was quenched in various ways: dry ice-methanol $\left(-73^{\circ} \mathrm{C}\right)$, ice-water $\left(0^{\circ} \mathrm{C}\right)$, water $\left(26^{\circ} \mathrm{C}\right)$, or put into annealing box set at different crystallization temperatures $\left(60^{\circ}-140\right.$ $\left.{ }^{\circ} \mathrm{C}\right)$. Neat PP specimens with same thermal histories were prepared as control samples.

Tensile stress-stain curves were measured at room temperature with a tensile testing machine, Tensilon UTM-II-20 (Toyo Baldwin Co. Ltd.) with a crosshead speed of $2 \mathrm{~mm} \mathrm{m^{-1 }}$. After the pre-set strain was attained, the crosshead returned at the same speed as stretching. After the strain reached zero, the sample was released from the clamps and residual strain was measured at certain intervals.

For transmission electron microscopy (TEM), the specimens were stained with ruthenium tetroxide $\left(\mathrm{RuO}_{4}\right)$ vapour for $30 \mathrm{~min}$ at $50^{\circ} \mathrm{C}$. The stained specimen was microtomed to obtain an ultrathin sections of $c a .70 \mathrm{~nm}$ thickness using an ultra-cryomicrotome (Ultracut N: Reichert-Nissei) at $-70^{\circ} \mathrm{C}$. The structure in section was observed with a JEOL electron microscope, JEM-100 CX.

In a differential scanning calorimeter (Seiko Instruments-EXSTAR 6000), the specimens were heated in nitrogen atmosphere. In case of linear temperature increase the rate was $10^{\circ} \mathrm{C} \mathrm{min}^{-1}$. The melting temperature was obtained from the minimum and enthalpy of fusion from the area of the endothermic peak. Crystallinity, $X_{\mathrm{c}}$, was calculated by $X_{\mathrm{c}}=\Delta H^{*} / \Delta H_{\mathrm{PP}}^{0}$, where $\Delta H^{*}$ is the enthalpy of fusion per gram of PP or that in the blend and $\Delta H_{\mathrm{PP}}^{\circ}$ is the heat of fusion per gram of $100 \%$ crystalline PP $\left(=209 \mathrm{~J} \mathrm{~g}^{-1}\right) .{ }^{11}$ In case of TMDSC (temperature modulated differential scanning calorimetry) experiment, ${ }^{12}$ the temperature increased in sinus function; $T=T_{0}+b t+B \sin (\omega t)$, where $T_{0}$ is the starting temperature $\left(100^{\circ} \mathrm{C}\right), t$ time, $b$ heating rate $\left(2^{\circ} \mathrm{C} \mathrm{min}-1\right)$, $\omega$ frequency $(0.04 \mathrm{~Hz})$ and $B$ amplitude $\left(0.5^{\circ} \mathrm{C}\right)$. Fourier transform treatment of the measured response was used for data deconvolution.

WAXD pattern was observed by Rigaku Denki RU-200 $\mathrm{X}$-Ray diffraction apparatus using scintillation counter system. Radiation from the $\mathrm{Cu}$ anode was reflected from a graphite monochromator to obtain monochromatic $\mathrm{Cu}$ $K_{\alpha}$ radiation with a wavelength of $0.1541 \mathrm{~nm}$. The generator was operated at $50 \mathrm{kV}$ and $180 \mathrm{~mA}$.

\section{RESULTS AND DISCUSSION}

Figure 1 shows the stress-strain behavior of $50 / 50$ and $25 / 75 \mathrm{PP} / \mathrm{hBR}$ blends. The blends were stretched to $50 \%$ elongation and then the movement of clamp was reversed at the same speed. The inserted small picture shows the curve for neat PP. Even though the temperature of quenching medium was very low $\left(-73^{\circ} \mathrm{C}\right)$, the

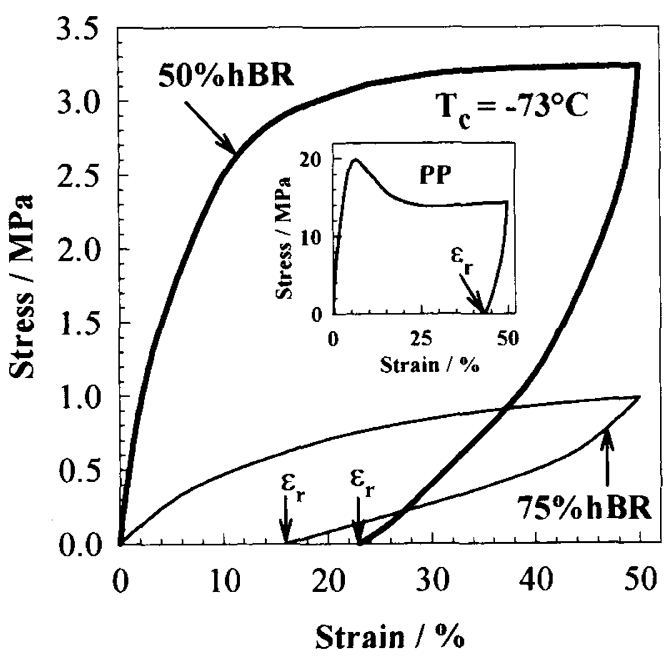

Figure 1. Typical stress-strain curves for quenched blends in comparison with pure PP.

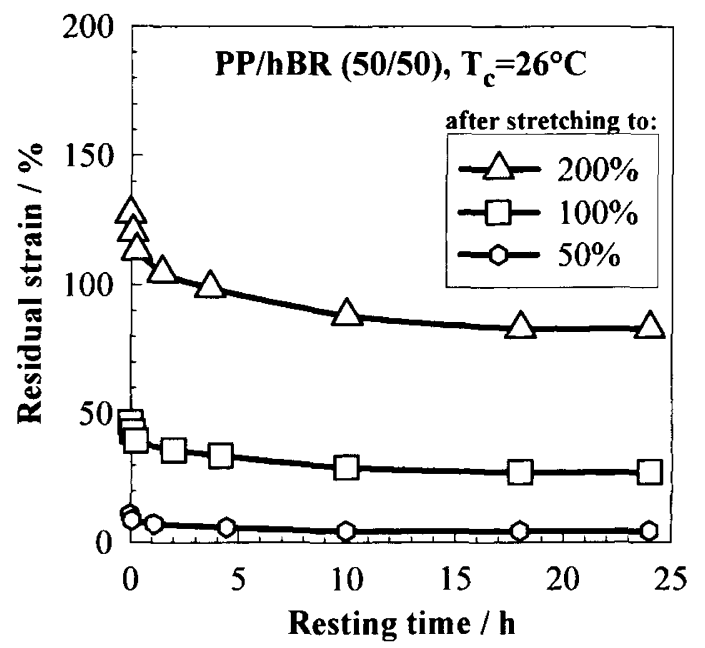

Figure 2. Residual strain after release from 200, 100, or 50\%elongation as function of resting time.

curve for PP shows clear yielding behavior with plastic deformation starting at about $8 \%$-elongation. The residual strain of the neat PP is very high (43\%), suggesting big plastic deformation. For immiscible blends with PP, similar curves with yielding point were obtained. ${ }^{13}$ In contrast, for blends with miscible hBR, there is no yielding point and residual strain $\varepsilon_{\mathrm{r}}$ is much smaller compared to neat PP. Residual strain decreases with resting time at room temperature, as shown in Figure 2.

In Figure 2 the residual strain is plotted as it was measured at certain time intervals. Steep recovery occurs during the first hour of resting, and then the recovery speed decreases. After $24 \mathrm{~h}$, the value did not change any more. Hence, the value of residual strain after $24 \mathrm{~h}$ should be compared for various blend compositions, applied strains and crystallization temperatures. Note that the recovery of neat $\mathrm{PP}$ was quite small (from 175 to $170 \%$ during $24 \mathrm{~h}$, after stretching to $200 \%$ ).

The influence of hBR content on residual strain is shown in Figure 3. The lowest residual strain values were found for blends containing 50 and $75 \%$ hBR after stretching to $50 \%$-elongation. $\varepsilon_{\mathrm{r}}$ was about 4 and $2 \%$, re- 


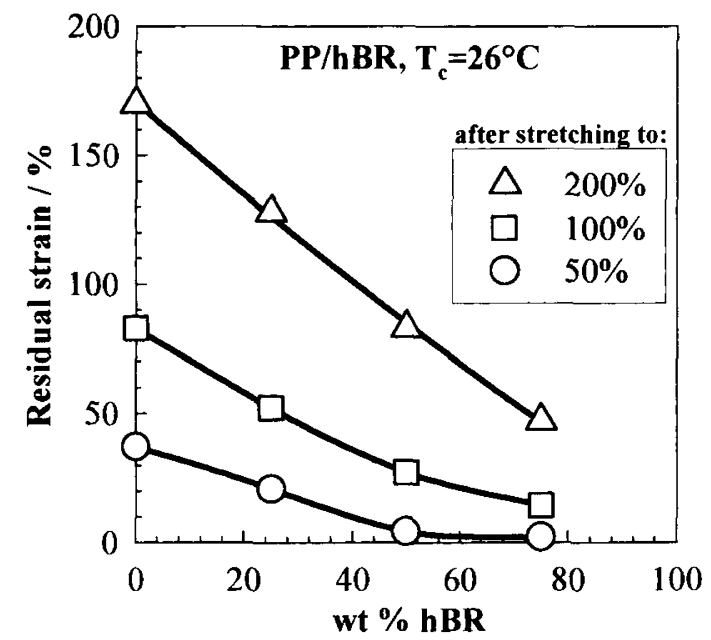

Figure 3. Residual strain vs. blend composition.

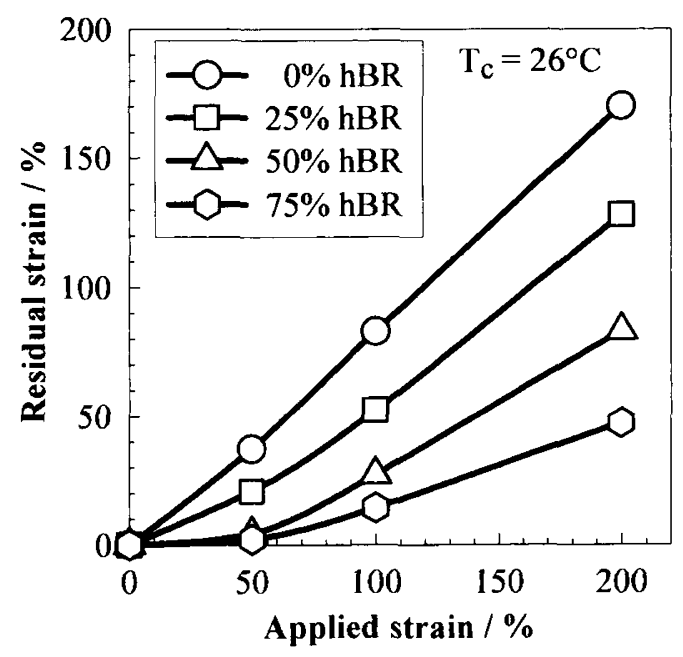

Figure 4. Applied strain $v s$. residual strain for samples quenched to $26^{\circ} \mathrm{C}$.

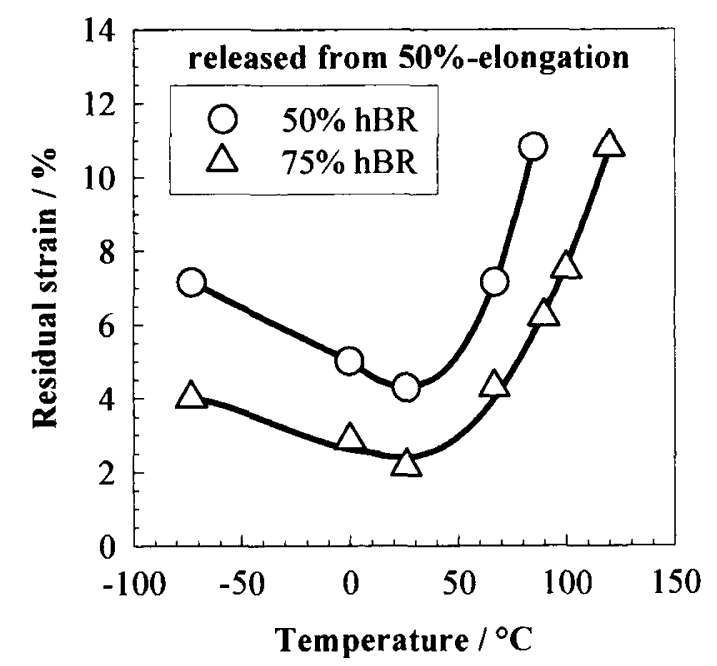

Figure 5. Residual strain $v s$. crystallization temperature $T_{\mathrm{c}}$ for two blend compositions.

spectively.

Residual strain is plotted as a function of applied strain for blends with different blend compositions in Figure 4. (a)

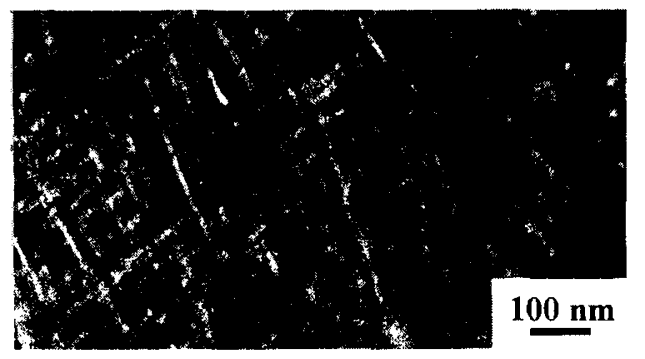

(b)

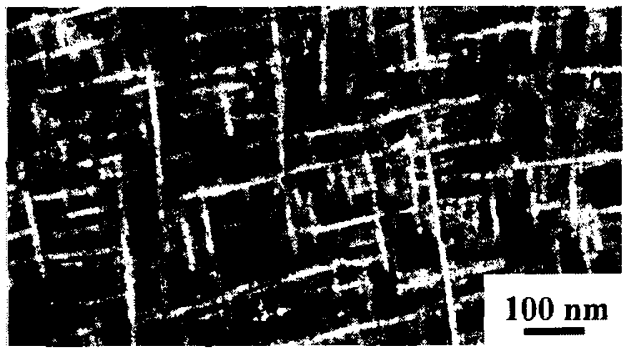

(c)

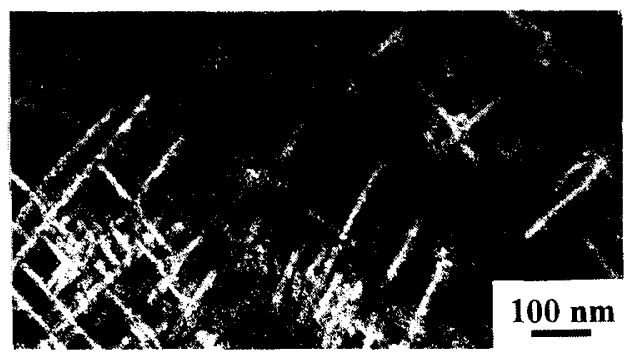

(d)

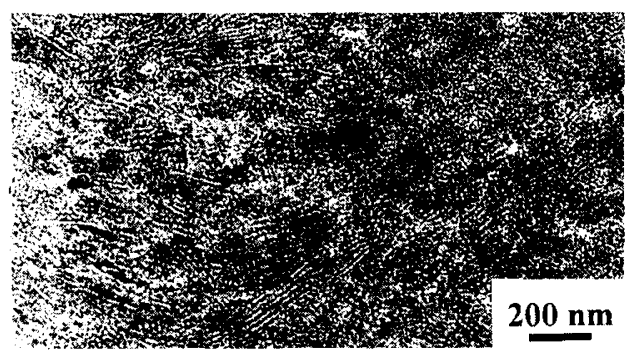

Figure 6. (a) - (c) TEM micrographs of $\mathrm{PP} / \mathrm{hBR}(25 / 75)$ blends crystallized at high temperatures: (a) $100^{\circ} \mathrm{C}$, (b) $120^{\circ} \mathrm{C}$, and (c) 140 ${ }^{\circ} \mathrm{C}$; (d) neat $\mathrm{PP}$ crystallized at $140^{\circ} \mathrm{C}$.

Figure 5 shows optimum crystallization condition to get best elastic properties. The results of two blends stretched to 50\%-elongation are shown. Optimum temperature of cooling medium is around room temperature. Crystallization at high temperatures is very harmful for elasticity. When the blend is crystallized at very low temperatures $\left(-73^{\circ} \mathrm{C}\right)$, elasticity gets a little bit worse, but the blend still remains elastic.

It is well known that the PP spherulite consists of crosshatched lamellae; i.e., subsidiary lamellae grow tangentially to the radiating primary lamellae. ${ }^{14}$ The fraction of subsidiary lamellae in PP spherulite is high at a low crystallization temperature $T_{\mathrm{C}}$, and decreases with $T_{\mathrm{C}}{ }^{15}$

Figure 6 shows three TEM micrographs $(a-c)$ of blend containing $75 \%$ of hBR crystallized at high temperatures. The crosshatch lamellar structure is seen. The lamellae are very long and thickness is about 10,12 , and $14 \mathrm{~nm}$ for crystallization temperatures 100,120 , and 140 ${ }^{\circ} \mathrm{C}$, respectively. For comparison, TEM of neat PP crystallized at $140^{\circ} \mathrm{C}$ is shown in Figure $6 \mathrm{~d}$. In contrast to the blends, lamellae prefer parallel alignment and are 
(a)

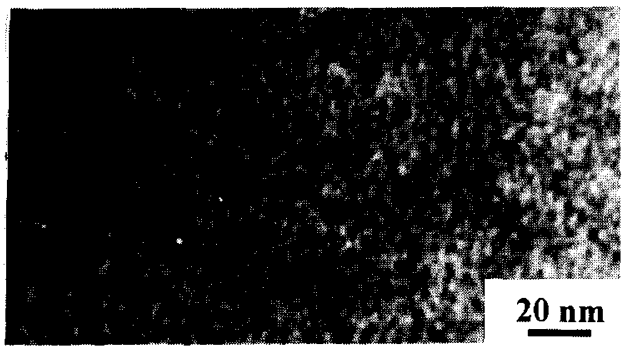

(b)

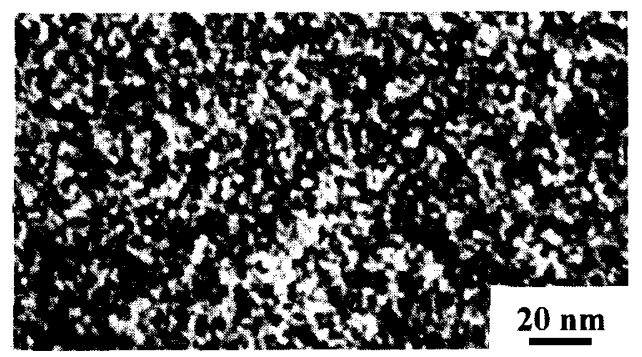

(c)

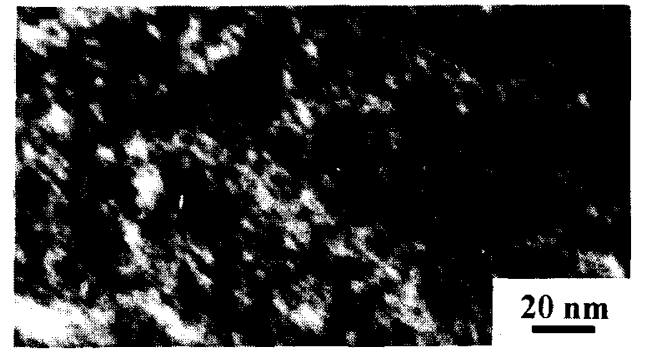

Figure 7. TEM micrographs of $\mathrm{PP} / \mathrm{hBR}(50 / 50)$ blends quenched to low temperatures: (a) $-73^{\circ} \mathrm{C}$, (b) $0^{\circ} \mathrm{C}$, and (c) $26^{\circ} \mathrm{C}$.

Table I. Melting point $T_{\mathrm{m}}$, crystallinity $X_{\mathrm{c}}$, apparent crystal size $D$ and morphology as function of crystallization temperature $T_{\mathrm{c}}$; obtained by DSC, WAXD, and TEM analyses

\begin{tabular}{cccrl}
\hline$T_{\mathrm{c}}{ }^{\circ} \mathrm{C}$ & $T_{\mathrm{m}} /{ }^{\circ} \mathrm{C}$ & $X_{\mathrm{c}}{ }^{\prime} \%$ & $D / \mathrm{nm}$ & Morphology \\
\hline-73 & 159.8 & 15.3 & 5.4 & Fragmented \\
0 & 161.3 & 18.3 & 10.0 & Fragmented \\
26 & 161.5 & 21.8 & 13.9 & Fragmented \\
140 & 164.2 & 28.0 & 15.0 & Crosshatch \\
\hline $0^{\circ} \mathrm{C}+$ annealing at $140^{\circ} \mathrm{C}$ & 161.7 & 22.8 & 12.7 & Fragmented \\
\hline
\end{tabular}

very close together. The well-developed crosshatch lamellar structure may be harmful for good elastic properties, because by bulk deformation a plastic deformation of the lamellae would occur.

Figure 7 shows three TEM micrographs of blends containing $50 \%$ of hBR quenched to low temperatures. There are no long crosshatch lamellae but only round particles (bright domains). The round particles could be assigned to PP crystallites, since the crystallites should be less stained by $\mathrm{RuO}_{4}$. Diffraction peaks of WAXD from the blend (see Figure 11) appeared at exactly the same diffraction angles as for neat PP. The melting temperatures $T_{\mathrm{m}}$ by DSC were very close to $T_{\mathrm{m}}$ of neat PP (Table I). These results clearly support that even in the blends quenched to very low temperatures, PP crystals do exist, and are observed as round particles under TEM. From the broadness of WAXD profile, apparent crystal size (D) may be estimated by the Scherrer equation. ${ }^{16}$ Crystal size in the perpendicular direction to the [110] plane was estimated from a peak at $2 \theta=14.16^{\circ}$.

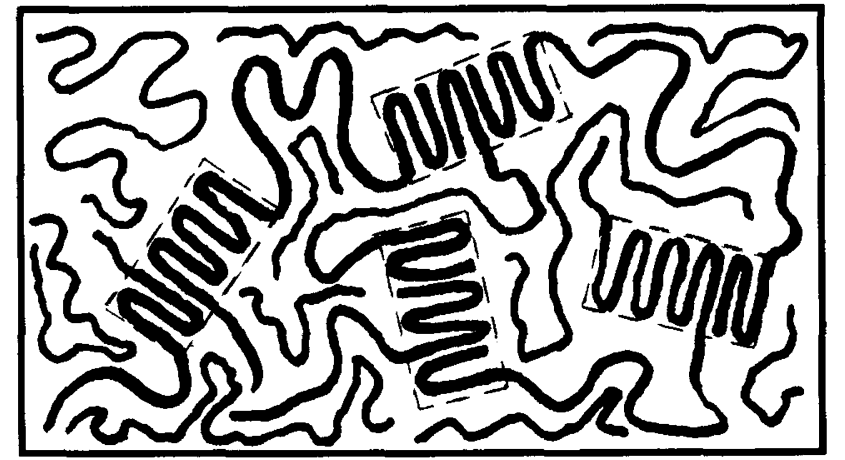

Figure 8. Schematic picture of the fragmented lamellar crystallites.

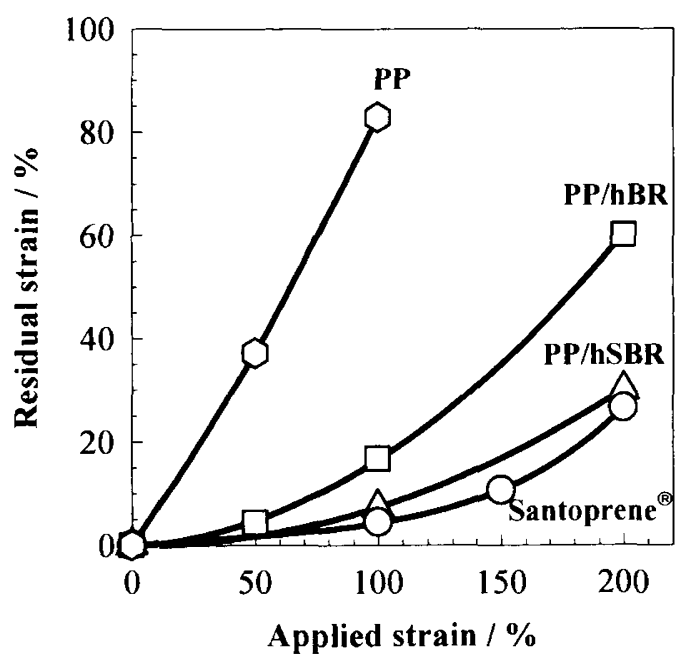

Figure 9. Comparison of elastic behavior of Santoprene, ${ }^{(k)} 850 / 50$ PP/hSBR blend, ${ }^{10} 50 / 50 \mathrm{PP} / \mathrm{hBR}$ blend, and neat PP.

Apparent crystal size in the blend quenched to low temperature $\left(-73^{\circ} \mathrm{C}\right.$ and $\left.0^{\circ} \mathrm{C}\right)$ is quite small. This is consistent with TEM observation (Figures $7 \mathrm{a}$ and $7 \mathrm{~b}$ ). Then, we call them "fragmented lamellar crystallites", because crystal growth to big lamellar crystals would be suppressed by the presence of high molecular weight impurity (hBR). The structures of fragmented lamellar crystallites may be depicted as in Figure 8. The fragmented lamellar crystallites may function as tie points for amorphous chains to provide a good elastic behavior.

When the blend was quenched to room temperature (Figure 7c), crystal size was slightly bigger. For this blend, optimum elastic properties were found. From results in Figures 5-7 and Table I, one can conclude that there is an appropriate crystal size to achieve good elastic property; i.e., when crystals are too small or too large, it is not good for elastic behavior.

Finally, it is interesting to compare elastic behavior of $\mathrm{PP} / \mathrm{hBR}$ blend with that of the PP/hSBR blend which contains non-crosslinked rubber particles of size $20 \mathrm{~nm}$, and with that of a commercial TPE Santoprene ${ }^{\circledR}$, which contains crosslinked rubber particles $1-3 \mu \mathrm{m}$. The comparison is shown in Figure 9. The PP/hSBR blend and Santoprene ${ }^{\mathbb{R}}$ have better elastic behavior than $\mathrm{PP} / \mathrm{hBR}$; i.e., by the presence of rubber particles the elastic behavior seems to be improved. The favourable contribution of rubber particles to elastic behavior can be due to volumetric strain of the rubber phase with high Poisson's ra- 


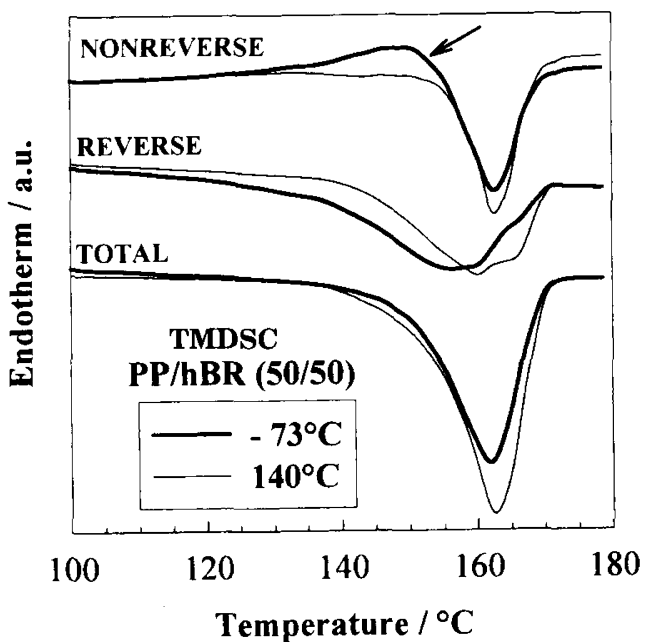

Figure 10. TMDSC of $\mathrm{PP} / \mathrm{hBR}$ (50/50) blends crystallized at $-73^{\circ} \mathrm{C}$ and $140^{\circ} \mathrm{C}$.

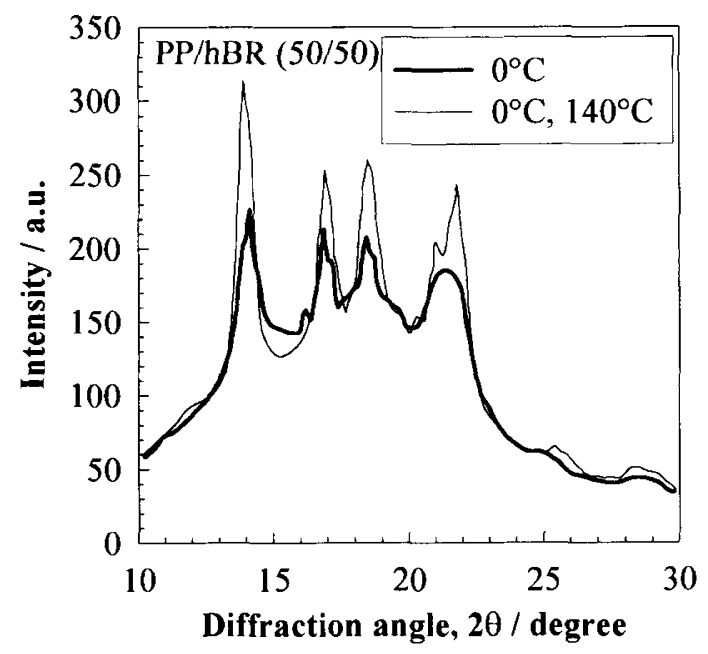

Figure 11. WAXD curves of $\mathrm{PP} / \mathrm{hBR}(50 / 50)$ blends.

tio ( $\sim 0.5$; very small volumetric change with bulk deformation), as has been discussed by finite element measurement (FEM) analysis. ${ }^{17}$ However, one can understand that the contribution is minor, if one compares with the strain recovery of neat PP. Major contribution is from the elastic nature of matrix in PP/hSBR and Santoprene ${ }^{\mathbb{R}}$, which originates from the characteristic morphology of "fragmented PP crystallites" as in the case of PP/hBR blend.

\section{APENDIX}

More fragmented crystallites formed by quenching to very low temperatures $\left(0^{\circ} \mathrm{C},-73^{\circ} \mathrm{C}\right)$ render less elastic behavior (see Figure 5 and Table I). The less ordered crystallites thus may not play the role of tie points, since they easily undergo plastic-deformation by bulk deformation. If crystallites get more ordered state by thermal annealing, elastic properties may improve.

Figure 10 shows the results of TMDSC analysis for samples crystallized at $140^{\circ} \mathrm{C}$ and $-73^{\circ} \mathrm{C}$. Conventional DSC showed curves similar to TOTAL curves in this Figure. In TOTAL curves, there is only difference in heat of fusion (area below the peak). On the NONREVERSE

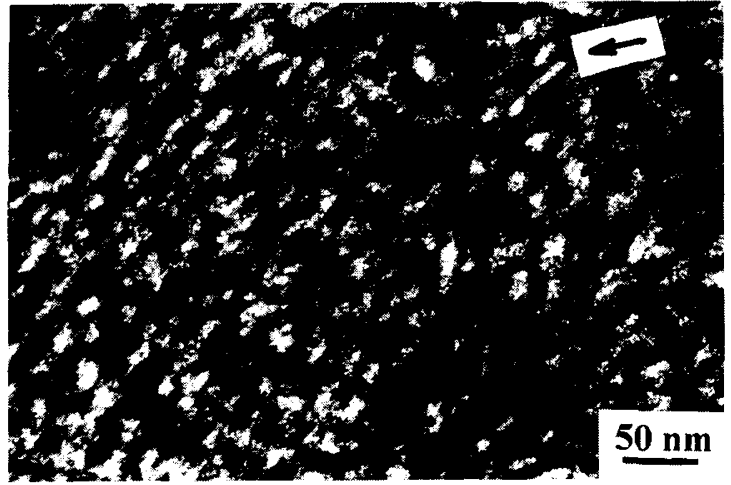

Figure 12. TEM micrograph of $\mathrm{PP} / \mathrm{hBR}(50 / 50)$ blend quenched to $0^{\circ} \mathrm{C}$ and then annealed $17 \mathrm{~h}$ at $140^{\circ} \mathrm{C}$.

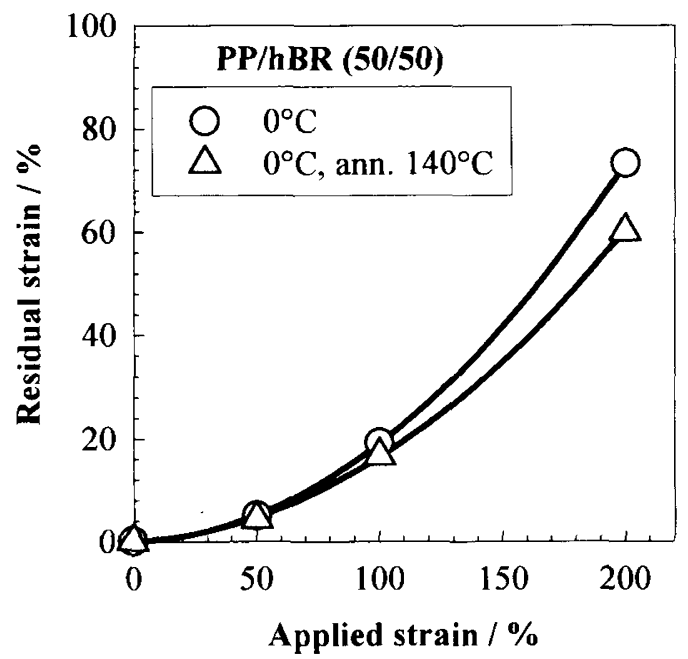

Figure 13. Residual strain vs. applied strain of PP/hBR (50/50) blends of blend quenched to $0^{\circ} \mathrm{C}$ in comparison with blend quenched to $0^{\circ} \mathrm{C}$ and then annealed to $140^{\circ} \mathrm{C}$.

curve the exothermic peak (shown by arrow) is seen for blend quenched to $-73^{\circ} \mathrm{C}$. It suggests that when the quenched blend was heated above $120^{\circ} \mathrm{C}$, recrystallization took place. The reference sample crystallized at 140 ${ }^{\circ} \mathrm{C}$ does not show any exothermic peak, implying that the well-crystallized lamellae had been formed. Recrystallization in the blend quenched to $-73^{\circ} \mathrm{C}$ may involve healing of defects and increase in crystal size.

To investigate the effects of recrystallization on crystal structure and elastomeric property, a 50/50 blend was first quenched to $0^{\circ} \mathrm{C}$, and annealed for $17 \mathrm{~h}$ at 140 ${ }^{\circ} \mathrm{C}$. In Figure 11 WAXD curves before and after the annealing are shown. With annealing, crystallinity increased from 18.3 to $22.8 \%$ and apparent crystal size increased from 10.0 to $12.7 \mathrm{~nm}$. The structure of the blend after annealing by TEM is shown in Figure 12. At the upper right, there is a white stripe of the size approximately $7 \times 35 \mathrm{~nm}$ (shown by arrow), showing clearly that the size of fragmented lamellae increased with annealing. TEM also shows that fragmented character of lamellae still remains even after annealing. The fragmented lamellae thus cannot grow to crosshatch lamellae as in the case of neat PP. Figure 13 shows the strain recovery behavior of the blend before and after annealing. There is a small improvement possibly caused by ordering in crystal structure. 


\section{CONCLUSION}

$\mathrm{PP} / \mathrm{hBR}$ blends served as a model for explanation of the elastic behavior of polyolefinic TPE containing PP. There is a big difference in structure and properties for neat PP and PP/hBR blend. Regardless of crystallization temperature, PP shows always yielding point, i.e., plastic deformation after stretching even to small deformation. In neat PP, crosshatch lamellar crystals were always formed. For PP/hBR blend, elastic properties are highly affected by crystallization temperature. Optimum in temperature of cooling medium was around room temperature. In this case the formation of long crosshatch lamellae was prevented and small fragmented crystal lamellae were formed. Fragmented crystallites function as tie points for amorphous chains to render good elastomeric behavior. There is no yielding point in stressstrain curves for those blends. Elastic behavior can be improved by annealing the blend at higher temperature (after quenching), when the crystal size increases, but the fragmented character remains. When the blend crystallizes at high temperature, large crosshatch lamellae are formed and the blend shows very poor elastic properties.

Acknowledgment. P. Svoboda thanks Japan Society for the Promotion of Science (JSPS) for supporting his stay at Tokyo Institute of Technology. Thanks also go to Mr. Atsuhito Someya for help with TMDSC analysis.

\section{REFERENCES}

1. A. Y. Coran, in "Handbook of Elastomers-New Developments and Technology", A. K. Bhomwick and H. L. Stephens, Ed., Marcel Dekker, New York, N.Y., 1988.

2. A. Y. Coran and P. Patel, Rubber Chem. Technol., 53, 141 (1980).

3. Y. Kikuchi, T. Fukui, T. Okada, and T. Inoue, Polymer, 31, 1029 (1991).

4. J. D. Katsaros, M. F. Malone, and H. H. Winter, Polym. Eng. Sci., 29, 1434 (1989).

5. L. P. Rectpr, K. A. Mazich, and S. H. Carr, J. Macromol. Sci. Phys., B27, 421 (1988).

6. F. B. Cheikh Larbe, M. F. Malone, H. H. Winter, J. L. Halary, M. H. Leviet, and L. Monnerie, Macromolecules, 21, 3532 (1988).

7. H. Sano, H. Yui, H. Li, and T. Inoue, Polymer, 39, 5265 (1998).

8. Y. Yang, T. Chiba, H. Saito, and T. Inoue, Polymer, 39, 3365 (1998).

9. N. Otsuka, Y. Yang, H. Saito, T. Inoue, and Y. Takemura, Polymer, 39, 1533 (1998).

10. Y. Yang, N. Otsuka, H. Saito, T. Inoue, and Y. Takemura, Polymer, 40, 559 (1999).

11. S. Brandup and E. M. Immergut, Ed., "Polymer Handbook", Interscience, New York, N.Y., 1975.

12. M. Reading, D. Elliot, and V. L. Hill, J. Thermal Anal., 40, 949 (1993).

13. M. T. Payne and C. P. Rader, in "Elastomer Technology Handbook", N. P. Cheremisinof, Ed., CRC Press, Boca Raton, FL, 1993, Chapter 14.

14. F. J. Padden and H. D. Keith, J. Appl. Phys., 30, 1479 (1959).

15. B. R. Norton and A. Keller, Polymer, 26, 704 (1985).

16. B. Wunderlich, "Macromolecular Physics", Academic Press, New York, N.Y., 1973.

17. M. Okamoto, K. Shiomi, and T. Inoue, Polymer, 35, 4618 (1994). 